\title{
Modelling of the dust release from bulk solids in the event of particle impact through enhanced dust detachment functions
}

\author{
D. Schulz*1 ${ }^{* 1}$ M. Kaul ${ }^{2}$, G. Reznik ${ }^{2}$, E. Schmidt ${ }^{2}$ and H. Kruggel-Emden ${ }^{1}$ \\ ${ }^{1}$ Chair of Mechanical Process Engineering and Solids Processing \\ Technische Universität Berlin \\ Ernst-Reuter-Platz 1, D-10587 Berlin, Germany \\ E-mail: daniel.schulz@tu-berlin.de,Web page: https://www.mvta.tu-berlin.de/ \\ ${ }^{2}$ Institute of Particle Technology \\ University of Wuppertal \\ Rainer-Gruenter-Str, Geb. FF, D-42119 Wuppertal, Germany \\ Web page: https://www.ipt.uni-wuppertal.de/
}

\begin{abstract}
In terms of environmental, health and explosion protection, it is of the utmost importance to assess the extent of diffuse dust emissions. In addition to standardized dustiness tests and measurements under field conditions on real dust emissions, numerical methods such as the Discrete Element Method (DEM) coupled to Computational Fluid Dynamics (CFD) are promising approaches for the prediction of the latter [1]. Thereby the bulk solid is fully resembled in the DEM or DEM/CFD, while dust particles are usually not modelled in detail, but rather are described using so-called dust detachment functions, due to computing time restrictions. Approaches for describing the latter in the event of particle impact are e.g. the particle-based approach of Cui and Sommerfeld [2]. In our study, dust detachment functions are further developed based on a benchmarking with dust-resolved DEM simulations. Therefore, the adhesive contacts of fine dust particles attached to coarse bulk solid particles are modelled with a JKR based approach [3]. Depending on the velocity, impact angle, material properties and particle rotation in the case of single particle-wall and particle-particle contacts, a good match between dust-resolved DEM and DEM with integrated detachment functions can be provided. For the further derivation and verification of the method, it is planned to compare numerical results also to experimental investigations. Therefore, the fine dust particle amount is reproducibly applied to coarse bulk solid particles by a well-defined calcium carbonate powder. The adhesion of the powder phase is then analysed before and after an impact.
\end{abstract}

a)

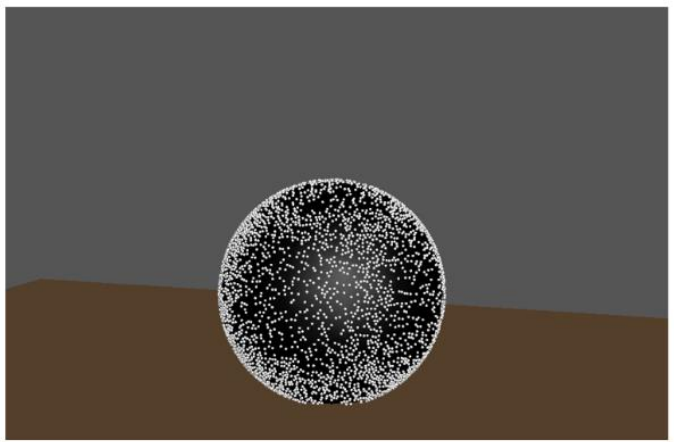

b)

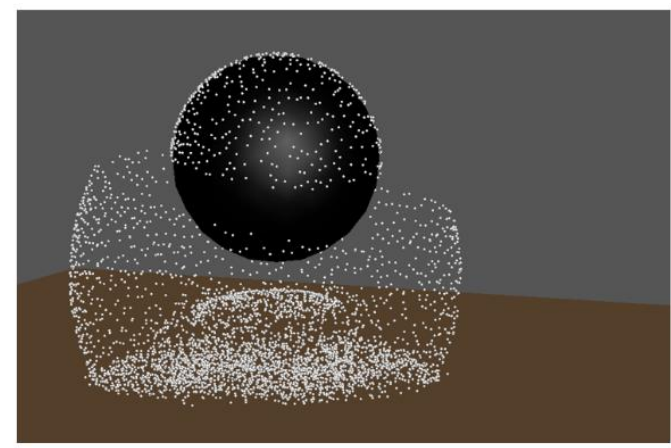

Fig. 1 Exemplary visualization of a DEM simulation a) before an impact and b) after an impact of a bulk solid particle.

\section{REFERENCES}

[1] S. Chakravarty, M. Fischer, O. Le, B. Martin, Towards a theoretical understanding of dustiness, Granul. Matter. (2019). doi:10.1007/s10035-019-0929-z.

[2] Y. Cui, M. Sommerfeld, The modelling of carrier-wall collision with drug particle detachment for dry powder inhaler applications, Powder Technol. 344 (2019) 741-755. doi:10.1016/j.powtec.2018.12.067.

[3] K.L. Johnson, K. Kendall, A.D. Roberts, Surface energy and the contact of elastic solids, in: Proc. R. Soc. Lond. A 324, 1971: pp. 301-313. 